\title{
Asymptotic Behavior of $M$-Band Scaling Functions of Daubechies Type
}

\author{
N. Bi, L. Debnath and Q. Sun
}

\begin{abstract}
This paper deals with the asymptotic behavior of $M$-band scaling functions ${ }_{N}^{M} \phi$ and $M$-band symbols $\stackrel{M}{N} H$ as $M \rightarrow \infty$ for $N \geq 2$. This is followed by pointwise convergence, and $L^{p}$-convergence $(1 \leq p<\infty)$ of $\underset{N}{M}$, and the limit function $g$ of $\underset{N}{N} \phi$ as $M \rightarrow \infty$.
\end{abstract}

Keywords: Wavelets, scaling functions, refinable functions

AMS subject classification: $\mathbf{4 2 C 1 5}$

\section{Introduction}

For any integer $M \geq 2$, a function $f$ is called $M$-refinable (or simply refinable) if it satisfies the refinement equation

$$
f(x)=\sum_{s \in \mathbf{Z}} c(s) f(M x-s)
$$

and $\int_{\mathbb{R}} f(x) d x=1$, where $\{c(s)\}$, called the mask of the refinement equation, satisfies the condition $\sum_{s \in \mathbf{Z}} c(s)=M$ and is of finite length. A function $f$ is said to be orthonormal if it satisfies

$$
\int_{\mathbb{R}} f(x) f(x-k) d x=\left\{\begin{array}{ll}
1 & \text { if } k=0 \\
0 & \text { if } k \neq 0
\end{array} \quad(k \in \mathbb{Z}) .\right.
$$

By a scaling function we mean an $M$-refinable and orthonormal function. For a given sequence $\{c(s)\}$, we define

$$
H(\xi)=\frac{1}{M} \sum_{s \in \mathbf{Z}} c(s) \exp (i s \xi)
$$

Then $H$ is called a filter of the refinement equation (1.1) or a filter corresponding to the scaling function $f$. For any integer $N \geq 1, H$ is said to have $N$ vanishing moments if there exists a Laurent polynomial $\tilde{H}$ such that

$$
H(z)=\left[\frac{1-z^{M}}{M(1-z)}\right]^{N} \tilde{H}(z) .
$$

Ning Bi: Hangzhou Normal College, Dept. Math., Hangzhou, Zhejiang 310012, China L. Debnath: University of Central Florida, Dept. Math., Orlando, Florida 32816, U.S.A. Qiyu Sun: Zhejiang University, Center for Math. Sci., Zhejiang 310027, China 
For a scaling function $f$, let a sequence of closed subspaces $V j \cdot(j \in \mathbb{Z})$ of square integrable function space $L^{2}(\mathbb{R})$ spanned by the functions

$$
f_{j, k}(x)=\left\{M^{j / 2} f\left(M^{j} x-k\right): k \in \mathbb{Z}\right\}
$$

Then $\left\{V_{j}\right\}_{j \in \mathbf{Z}}$ is called a multiresolution analysis of $L^{2}(\mathbb{R})$ if it satisfies the following conditions:

(i) $V_{j} \subset V_{j+1}$, and $f \in V_{j}$ if and only if $f(M x) \in V_{j+1}$ for all $j \in \mathbb{Z}$.

(ii) $\bigcup_{j \in Z} V_{j}$ is dense in $L^{2}(\mathbb{R})$ and $\bigcap_{j \in Z} V_{j}=\{0\}$.

(iii) $\{f(\cdot-k)\}_{k \in Z}$ is an orthonormal basis of $V_{0}$ for some of $f \in V_{0}$.

We denote the wavelet space $W_{j} \quad(j \in \mathbb{Z})$ by the orthonormal complement spaces of $V_{j}$ in $V_{j+1}$ so that the wavelet decomposition

$$
L^{2}=\bigcup_{l \in Z} W_{j}=V_{k}+\bigcup_{j \geq k} W_{j}
$$

holds. In fact, (1.5) suggests the decomposition

$$
f=\sum_{j \in \mathbf{Z}} g_{j}=\sum_{j \geq k} g_{j}+f_{k}
$$

of $f \in L^{2}(\mathbb{R})$ where $g_{j} \in W_{j}$ and $f_{k} \in V_{k}$.

The literature of wavelets is replete with analysis of 2-band ( $M=2$ ) scaling functions. The wavelet theory when $M=2 \mathrm{can}$ be found in the literature of wavelets (see Daubechies [2]). When $M=2, W_{j}$ is spanned by $\left\{2^{j} \psi\left(2^{j} \cdot-k\right)\right\}_{k \in \mathbf{Z}}$ and the mother wavelet can be constructed from the 2-band scaling functions $\phi$ in the form

$$
\psi(x)=\sum_{k \in Z} c_{1-k}(-1)^{k} \phi(2 x-k)
$$

where $c_{k}$ are the coefficient of the 2-band scaling functions defined by (1.1).

In short, the theory of wavelets for $M=2$ has received considerable attention. However, the wavelet theory for $M>2$ received much less attention. Bi et al. [1] and Heller [3] independently considered the design of filter with $N$ vanishing moment and finite length. Bi et al. [1] also considered $M$-band scaling functions, $M$-band wavelets and constructed compactly supported orthonormal $M$-band wavelets. The major objective of this paper is to investigate the asymptotic behavior of $M$-band scaling functions and $M$-band symbols as $M \rightarrow \infty$.

For any integer $N \geq 1$, let

$$
{ }_{N} H(\xi)=\frac{1}{2} \sum_{s=0}^{2 N-1} N a(s) \exp (i s \xi)
$$


be a solution of the equation

$$
\left.\left.\right|_{N} H(\xi)\right|^{2}=\cos ^{2 N}\left(\frac{\xi}{2}\right) \sum_{s=0}^{N-1}\left(\begin{array}{c}
2 N-1+s \\
s
\end{array}\right) \sin ^{2 s}\left(\frac{\xi}{2}\right)
$$

We note that the solution of equation (1.8) in the form

$$
{ }_{N} H(\xi)=\frac{1}{2} \sum_{s=0}^{2 N-1} N a(s) e^{i s \xi}
$$

is not unique, but finite when $N \geq 2$.

Daubechies [2] introduced scaling functions ${ }_{N} \phi$ with symbol ${ }_{N} H$ when $M=2$, and wavelets $N \psi$ defined by

$$
N \hat{\psi}(\xi)={ }_{N} H\left(-\frac{\xi}{2}+\pi\right) \exp \left(-\frac{i \xi}{2}\right){ }_{N} \hat{\phi}\left(\frac{\xi}{2}\right),
$$

where $\hat{f}$ is the Fourier transform of an integrable function $f$ defined by

$$
\hat{f}(\xi)=\int_{\mathbf{R}} \exp (-i x \xi) f(x) d x
$$

For these wavelets $N \psi,\left\{2_{N}^{j / 2} \psi\left(2^{j} \cdot-k\right)\right\}_{j, k \in \mathbf{Z}}$ is an orthonormal basis of $L^{2}(\mathbb{R})$. The Hölder index of $N \psi$ is about $\frac{\ln 3}{2 \ln 2} N$ for large $N$, and it has $N$ vanishing moments, that is,

$$
\int_{\mathbb{R}} x^{k}{ }_{N} \psi(x) d x=0 \quad(0 \leq k \leq N-1) .
$$

Moreover, for any $N \geq 1$, the scaling function $N \phi$ has minimal support in the class of compactly supported scaling functions $\phi$ for which we may find a compactly supported orthonormal wavelet $\psi$ in $V_{1}$ which has $N$ vanishing moments and satisfies

$$
\int_{\mathbb{R}} \psi(x) \phi(x-k) d x=0 \quad(k \in \mathbb{Z})
$$

where $V_{1}$ is the closed subspace of $L^{2}(\mathbb{R})$ spanned by $\{\sqrt{2} \phi(2 \cdot-k)\}_{k \in \mathbf{Z}}$.

We define

$$
\stackrel{M}{N} a(s)=\sum_{s_{1}+\ldots+s_{M-1}=s} \prod_{j=1}^{M-1}\left(\begin{array}{c}
N-1+s_{j} \\
s_{j}
\end{array}\right)\left(2 \sin \frac{j \pi}{M}\right)^{-2 s_{j}} \quad(0 \leq s \leq N-1)
$$

and

$$
P(t)=\sum_{s=0}^{N-1} \underset{N}{M} a(s) t^{s}
$$


By the Riesz lemma [2: p. 172/Lemma 6.1.3], there exists a unique solution $H$ of the equation

$$
|H(\xi)|^{2}=\left(\frac{\sin \frac{M \xi}{2}}{M \sin \frac{\xi}{2}}\right)^{2 N} P(2-2 \cos \xi),
$$

such that

$$
H(\xi)=\left(\frac{1-e^{i M \xi}}{M\left(1-e^{i \xi}\right)}\right)^{N} \sum_{s=0}^{N-1} \tilde{c}(s) e^{i s \xi}=\frac{1}{M} \sum_{s=0}^{M N-1} c(s) e^{i s \xi}
$$

and $\sum_{s=0}^{N-1} \tilde{c}(s) z^{s}$ has all roots in the open unit disk, where $P(z)$ is a polynomial in $z$. Denote the solution of equations (1.13) and (1.14) by ${ }_{N}^{M} H$. Let ${ }_{N}^{M} \phi$ be the solution of the refinement equation (1.1) with the symbol ${ }_{N}^{M} H$.

Bi et al. [1] and Heller [3] independently proved that ${ }_{N}^{M} \phi$ is orthonormal, and represents a scaling function. Furthermore; $\stackrel{M}{N} \phi$ has minimal support in the class of compactly supported scaling functions $\phi$ for which we may find compactly supported orthonormal wavelets $\psi_{s} \in V_{1} \quad(1 \leq s \leq M-1)$ such that $\psi_{\text {s }}$ has $N$-vanishing moments and $\left\{\phi(\cdot,-k), \psi_{s}(\cdot,-k)\right\}_{s ; k \in \mathbf{Z}}$ is an orthogonal basis of $V_{1}$, where $V_{1}$ is a closed subspace of $L^{2}$ spanned by $\left\{\sqrt{M} \phi(M \cdot-k\}_{k \in \mathbf{Z}}\right.$. For this reason, we call ${ }_{N}^{M} \phi$ as $M$-band scaling functions of Daubechies type.

When $M=2$, Daubechies [2] and Pollen [4] studied the 2-band scaling functions of Daubechies type. On the other hand, for $M$-band scaling functions of Daubechies type, $\mathrm{Bi}$ et al. [1] investigated the asymptotic bchavior of the Hölder index of ${ }_{N}^{M} \phi$ as $N \rightarrow \infty$. For $N=2$, Sun and Zhang [5] proved that the exact Hölder index of ${ }_{2}^{M_{\phi}}$ is $1-\frac{\ln (1+\theta)}{\ln M}$ where $\theta=\left\{\frac{1}{3}\left(2 M^{2}+1\right)\right\}^{1 / 2}$. The function ${ }_{2}^{M} \phi$ tends to a function $g$ pointwise as $M \rightarrow \infty$ where $g$ is given by

$$
g(x)=\left\{\begin{array}{ll}
x+\frac{\sqrt{6}}{6} & \text { if } 0<x \leq 1 \\
-x+1-\frac{\sqrt{6}}{6} & \text { if } 1<x \leq 2 \\
0 & \text { otherwise }
\end{array} .\right.
$$

They have also shown that ${ }_{2}^{M} \phi$ is locally linear on an open set with full measure and locally linearly dependent when $M \geq 3$.

This paper deals with studying the asymptotic behavior of $M$-band scaling functions ${ }_{N}^{M} \phi$ and $M$-band symbols ${ }_{N}^{M} H$ as $M \rightarrow \infty$, for any $N \geq 2$. More precisely, we investigate the local polynomial structure of ${ }_{N}^{M} \phi$ on an open set with full measure, the asymptotic behavior of ${ }_{N}^{M} H$, and then the pointwise convergence and $L^{p}$-convergence of ${ }_{N}^{M} \phi$ as $M \rightarrow \infty$. In Section 2, we consider the local polynomial structure of ${ }_{N}^{M} \phi$ on an open set. Section 3 deals with the asymptotic behavior of $M$-band symbols ${ }_{N}^{M} H$. This is followed by pointwise convergence and $L^{p}$-convergence $(1 \leq p<\infty)$ of ${ }_{N}^{M} \phi$. Finally, some remarks on the limit function $g$ of ${ }_{N}^{M} \phi$ as $M \rightarrow \infty$ are discussed. 


\section{Local polynomial functions}

We say that a function supported in $[a, b]$ is locally polynomial on an open set $A \subset[a, b]$ if it is a polynomial on every connected component of $A$.

Theorem 2.1. Let $M>N$ and ${ }_{N}^{M} \phi$ be the solution of the refinement equation (1.1) with symbol ${ }_{N}^{M} H$. Then there exists an open set $A \subset\left(0, N+\frac{N-1}{M-1}\right)$ with Lebesgue measure $N+\frac{N-1}{M-1}$ such that ${ }_{N}^{M} \phi$ is locally polynomial on $A$.

Moreover, the above assertion holds for a more general class of refinable functions. A proof of this theorem is given by $\mathrm{Bi}$ et al. [1], and is omitted.

Theorem 2.2. Let $M-1>r \neq 0$ and $\phi$ be the solution of the refinement equation (1.1) with symbol

$$
H(\xi)=\left(\frac{1-e^{i M \xi}}{M\left(1-e^{i \xi}\right)}\right)^{N} Q_{r}(\xi)
$$

where $Q_{r}(0)=1$ and $Q_{r}(\xi)$ may be written as $Q_{r}(\xi)=\sum_{k=0}^{r} c(k) e^{i k \xi}$. Then there exists an open set $A \subset\left(0, N+\frac{r}{M-1}\right)$ with Lebesgue measure $N+\frac{r}{M-1}$ such that $\phi$ is locally polynomial on $A$.

To prove Theorem 2.2 , we need some lemmas.

Let $\phi$ be as in Theorem 2.2. We define

$$
\begin{aligned}
& \Phi(x)=(\phi(x), \ldots, \phi(x+N-1))^{T} \\
& \widetilde{\Phi}(x)=(\phi(x+1), \ldots, \phi(x+N))^{T} \quad(x \in(0,1))
\end{aligned}
$$

and

$$
m_{j}=\int_{\mathbb{R}} x^{j} \phi(x) d x \quad(0 \leq j \leq N-1)
$$

Let

$$
A(x)=\left((x+k)^{j}\right)_{0 \leq j, k \leq N-1} \quad \text { and }: \quad \widetilde{A}(x)=\left((x+k)^{j}\right)_{\substack{0 \leq j \leq N-1 \\ i \leq k \leq N}}
$$

Denote the transpose of a matrix (or a vector) $A$ by $A^{T}$. Then we have the following

Lemma 2.1. Let $M-1>r$ and $\phi$ be as in Theorem 2.2. Then

$$
\left.\begin{array}{l}
A(x) \Phi(x)=\left(m_{0}, \ldots, m_{N-1}\right)^{T}-\left(1, x+N, \ldots,(x+N)^{N-1}\right)^{T} \phi(x+N) \\
\widetilde{A}(x) \widetilde{\Phi}(x)=\left(m_{0}, \ldots, m_{\dot{N}-1}\right)^{T}-\left(1 ; x, \ldots, x^{N-1}\right)^{T} \phi(x)
\end{array}\right\}
$$

on $(0,1)$ and $\phi$ is polynomial on $\bigcup_{j=0}^{N-1}\left(j+\left(\frac{r}{M-1}, 1\right)\right)$.

Proof. We first note that $\phi$ is supported on $\left[0, N+\frac{r}{M-1}\right]$ and

$$
\operatorname{det} A(x)=\prod_{0 \leq i<j \leq N-1}(j-i) \neq 0
$$


Therefore, from the first formula in (2.1) we get

$$
\Phi(x)=(\operatorname{det} A(x))^{-1} A^{*}(x)\left(m_{0}, \ldots, m_{N-1}\right)^{T}
$$

on $\left(\frac{r}{M-1}, 1\right)$, where $A^{*}(x)$ denotes the adjoint matrix of $A(x)$. Then the second assertion follows from (2.1).

Now we prove (2.1). By taking the Fourier transform of both sides of the refinement equation (1.1), we obtain

$$
\hat{\phi}(\xi)=H\left(\frac{\xi}{M}\right) \hat{\phi}\left(\frac{\xi}{M}\right) .
$$

Therefore, $D^{j} \hat{\phi}(2 k \pi)=0$ for all $k \in \mathbb{Z} \backslash\{0\}$ and $0 \leq j \leq N-1$, where $D=\frac{\partial}{\partial \xi}$ is a differential operator, and furthermore

$$
\sum_{k \in \mathbf{Z}}(x+k)^{j} \phi(x+k)=\int_{\mathbb{R}} x^{j} \phi(x) d x=m_{j} \quad(0 \leq j \leq N-1)
$$

by the Poisson summation formula. Then the first assertion in (2.1) follows from (2.3)

Lemma 2.2. Let $\phi$ be the same as in Theorem 2.2. Then there exist real numbers $a(0), \ldots, a(r)$ and polynomials $P_{1}, \ldots, P_{r}$ with degree at most $N-1$ such that

$$
\phi\left(\frac{x+j}{M}\right)=a(j) \phi(x)+P_{j}(x) \quad(0 \leq j \leq r, x \in(0,1))
$$

and

$$
\begin{aligned}
\phi\left(\sum_{j=1}^{k} \frac{\varepsilon_{j}}{M^{j}}+\frac{x}{M^{k}}\right)= & \prod_{j=1}^{k} a\left(\varepsilon_{j}\right) \phi(x)+P_{\varepsilon_{k}}\left(\sum_{j=2}^{k} \frac{\varepsilon_{j}}{M^{j-1}}+\frac{x}{M^{k-1}}\right) \\
& +\sum_{i=0}^{k-2} \prod_{l=k-i}^{k} a\left(\varepsilon_{l}\right) P_{\varepsilon_{k-1-i}}\left(\sum_{j=i+3}^{k} \frac{\varepsilon_{j}}{M^{j-i-2}}+\frac{x}{M^{k-i-2}}\right)
\end{aligned}
$$

where $\varepsilon_{j} \in\{0,1 \ldots, r\}$ and $x \in(0,1)$.

Proof. By the refinement equation (1.1), we obtain

$$
\phi\left(\frac{x+j}{M}\right)=\sum_{l=0}^{(M-1) N+r} c_{l} \phi(x+j-l)=\sum_{l=0}^{j} c_{j-l} \phi(x+l)
$$

on $(0,1)$. From Lemma 2.1, there exist polynomials $Q_{j} \in \Pi_{N-1}$ and numbers $d_{j} \quad(1 \leq$ $j \leq N)$ such that

$$
\phi(x+j)=d_{j} \dot{\phi(x)}+Q_{j}(x)
$$

where $\Pi_{N-1}$ denotes the class of polynomials with degrees at most $N-1$. Then (2.4) follows from (2.6) and (2.7), and (2.5) follows by using formula (2.4) $k$ times 
For any $\varepsilon_{i} \in\{0,1, \ldots, r\}$ and $1 \leq i \leq k$, define

$$
A\left(\varepsilon_{1}, \ldots, \varepsilon_{k}\right)=\left(\sum_{j=1}^{k} \frac{\varepsilon_{j}}{M^{j}}+\frac{r}{(M-1) M^{k}}, \sum_{j=1}^{k} \frac{\varepsilon_{j}}{M^{j}}+\frac{1}{M^{k}}\right) .
$$

Then $A\left(\varepsilon_{1}, \ldots, \varepsilon_{k}\right) \subset\left(0, \frac{r}{M-1}\right)$ when $\varepsilon_{k} \neq r$. Furthermore, we have the following

Lemma 2.3. Let $A\left(\varepsilon_{1}, \ldots, \varepsilon_{k}\right)$ be defined as above. Then

$$
A\left(\varepsilon_{1}, \ldots, \varepsilon_{k}\right) \cap A\left(\varepsilon_{1}^{\prime}, \ldots, \varepsilon_{k^{\prime}}^{\prime}\right)=\emptyset
$$

when $\varepsilon_{k}, \varepsilon_{k^{\prime}}^{\prime} \neq r$ except $k=k^{\prime}$ and $\left(\varepsilon_{1}, \ldots, \varepsilon_{k}\right)=\left(\varepsilon_{1}^{\prime}, \ldots, \varepsilon_{k^{\prime}}^{\prime}\right)$.

Proof. Define

$$
a\left(\varepsilon_{1}, \ldots, \varepsilon_{k}\right)=\sum_{j=1}^{k} \frac{\varepsilon_{j}}{M^{j}}+\frac{r}{(M-1) M^{k}} \quad \text { and } \quad b\left(\varepsilon_{1}, \ldots, \varepsilon_{k}\right)=\sum_{j=1}^{k} \frac{\varepsilon_{j}}{M^{j}}+\frac{1}{M^{k}} .
$$

Then it suffices to prove that

$$
a\left(\varepsilon_{1}^{\prime}, \ldots, \varepsilon_{k^{\prime}}^{\prime}\right)>a\left(\varepsilon_{1}, \ldots, \varepsilon_{k}\right) \quad \Longrightarrow \quad a\left(\varepsilon_{1}^{\prime}, \ldots, \varepsilon_{k^{\prime}}^{\prime}\right) \geq b\left(\varepsilon_{1}, \ldots, \varepsilon_{k}\right)
$$

We note that

$$
M^{j} a\left(\varepsilon_{1}, \ldots, \varepsilon_{k}\right)=M^{j} \sum_{j=1}^{j} \frac{\varepsilon_{i}}{M^{i}}+M^{j} a\left(\varepsilon_{j+1}, \ldots, \varepsilon_{k}\right) \subset M^{j} \sum_{j=1}^{j} \frac{\varepsilon_{i}}{M^{i}}+(0,1)
$$

and

$$
M^{j} b\left(\varepsilon_{1}, \ldots, \varepsilon_{k}\right)=M^{j} \sum_{j=1}^{j} \frac{\varepsilon_{i}}{M^{i}}+M^{j} b\left(\varepsilon_{j+1}, \ldots, \varepsilon_{k}\right) \subset M^{j} \sum_{j=1}^{j} \frac{\varepsilon_{i}}{M^{i}}+(0,1] .
$$

Therefore the problem reduces to prove

$$
b\left(\varepsilon_{1}, \ldots, \varepsilon_{k}\right) \leq a\left(\varepsilon_{1}^{\prime}, \ldots, \varepsilon_{k^{\prime}}^{\prime}\right)
$$

for the following two cases: (i) $\varepsilon_{1}^{\prime} \neq \varepsilon_{1}$ and (ii) $\varepsilon_{1}=\varepsilon_{1}^{\prime}$ and $k=1$ or $k^{\prime}=1$.

For the case (i), we get $\varepsilon_{1}^{\prime}>\varepsilon_{1}$, otherwise

$$
a\left(\varepsilon_{1}^{\prime}, \ldots, \varepsilon_{k^{\prime}}^{\prime}\right)<\frac{\varepsilon_{1}^{\prime}+1}{M} \leq a\left(\varepsilon_{1}, \ldots, \varepsilon_{k}\right)
$$

which is a contradiction. Therefore, we have

$$
b\left(\varepsilon_{1}, \ldots, \varepsilon_{k}\right) \leq \frac{\varepsilon_{1}+1}{M} \leq a\left(\varepsilon_{1}^{\prime}, \ldots, \varepsilon_{k^{\prime}}^{\prime}\right) .
$$

For the case (ii), $k^{\prime}$ must be one, otherwise

$$
a\left(\varepsilon_{1}^{\prime}, \ldots, \varepsilon_{k}^{\prime}\right)<\frac{\varepsilon_{1}}{M}+\sum_{j=2}^{k} \frac{r}{M^{j}}+\frac{r}{(M-1) M^{k}}=\frac{\varepsilon_{1}}{M}+\frac{r}{(M-1) M}=a\left(\varepsilon_{1}\right)
$$

which is a contradiction. Therefore, we have

$$
b\left(\varepsilon_{1} \ldots, \varepsilon_{k}\right) \leq \frac{\varepsilon_{1}}{M}+\sum_{j=\dot{2}}^{k-1} \frac{r}{M^{j}}+\frac{r-1}{M^{k}}+\frac{1}{M^{k}} \leq a\left(\varepsilon_{1}^{\prime}\right)
$$

and the lemma is proved 
Proof of Theorem 2.2. We define

$$
O=\bigcup_{k=1}^{\infty} \bigcup_{\substack{\left(\varepsilon_{1}, \ldots, \varepsilon_{k}-1\right) \in(0,1, \ldots, r)^{k-1} \\ \varepsilon_{k} \in(0,1 \ldots, r-1)}} A\left(\varepsilon_{1}, \ldots, \varepsilon_{k}\right)
$$

and

$$
A=\left(\bigcup_{i=0}^{N}(O+i)\right) \bigcup\left(\bigcup_{i=0}^{N-1}\left(i+\left(\frac{r}{M-1}, 1\right)\right)\right) .
$$

Then $\phi$ is local polynomial on $A$ by Lemmas 2.1 and 2.2. By Lemma 2.3, we obtain

$$
\begin{aligned}
& |A|=(N-1)\left(1-\frac{r}{M-1}\right)+N \sum_{k=1}^{\infty} \sum_{\substack{\left(\varepsilon_{1}, \ldots, \varepsilon_{k-1}\right) \in\left\{(0,1, \ldots, r\}^{k-1} \\
\varepsilon \in k(0,1, \ldots, r-1\}\right.}}\left|A\left(\varepsilon_{1}, \ldots, \varepsilon_{k}\right)\right| \\
& =(N-1)\left(1-\frac{r}{M-1}\right)+N \sum_{k=1}^{\infty}\left(1-\frac{r}{M-1}\right) \frac{r}{M}\left(\frac{r+1}{M}\right)^{k-1} \\
& =N+\frac{r}{M-1} \text {. }
\end{aligned}
$$

This proves the theorem

\section{Asymptotic behavior of $M$-band symbol}

We write

$$
{ }_{N}^{M} H(\xi)=\left(\frac{1-e^{i M \xi}}{M\left(1-e^{i \xi}\right)}\right)^{N}{ }_{N}^{M} \widetilde{H}(\xi)
$$

and

$$
{ }_{N}^{M} \tilde{H}(\xi)=\sum_{s=0}^{N-1} a_{M}(s)\left(e^{i \xi}-1\right)^{s} .
$$

Then we have the following

Theorem 3.1. Let ${ }_{N}^{M} \widetilde{H}(\xi)$ be defined by (3.2). Then $a_{M}(0)=1$ and the limit of $a_{M}(s) M^{-s}$ exists for $1 \leq s \leq N-1$ and

$$
\lim _{M \rightarrow \infty}{ }_{N}^{M} H\left(\frac{\xi}{M}\right)=\left(\frac{1-e^{i \xi}}{-i \xi}\right)^{N} \sum_{s=0}^{N-1} \alpha(s)(i \xi)^{s}
$$

where $\alpha(s)=\lim _{M \rightarrow \infty} a_{M}(s) M^{-s}(0 \leq s \leq N-1)$. Furthermore,

$$
\left|M^{-(N-1)} a_{M}(N-1)\right| \leq 2^{-N+1}\left(1-\left|\frac{2}{\pi}\right|^{2 N}\right)^{1 / 2}
$$


To prove Theorem 3.1, we need some lemmas.

Define

$$
A(k, s)=\sum_{l=0}^{s}\left(\begin{array}{c}
2 N-1+l \\
l
\end{array}\right)(2 k \pi)^{-2 l} A(k-1, s-l) \quad(k \geq 2)
$$

and

$$
A(1, s)=\left(\begin{array}{c}
2 N-1+s \\
s
\end{array}\right)(2 \pi)^{-2 s}
$$

Then $A(k, s) \geq A(k-1, s)$ and $|A(k, s)-A(k-1, s)| \leq C_{,} k^{-2}$ holds for all $k \geq 2$, where $C$, is a constant depending on $s$ only. Therefore, $\lim _{k \rightarrow \infty} A(k, s)$ exists for all $0 \leq s \leq N-1$. Denote its limit by $A_{s} \quad(0 \leq s \leq N-1)$ (the explicit computation of $A$, will be given in Section 5 ). Then we have the following:

Lemma 3.1. Let ${ }_{N}^{M} a(s)$ be defined by (1.6). Then

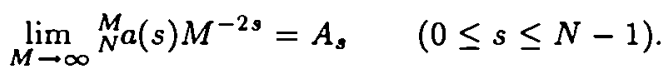

Proof. First we prove the assertion when $M$ is odd. Denote $M^{\prime}=\frac{M-1}{2}$. Then we may write

$$
\begin{aligned}
& \stackrel{M}{N}^{M} a(s)=M^{2 s} \sum_{s_{1}+\ldots+s_{M^{\prime}=s}}\left(\prod_{j=1}^{M^{\prime}}\left(\begin{array}{c}
2 N-1+s_{j} \\
s_{j}
\end{array}\right)(2 j \pi)^{-2 s_{j}}\right) \\
& \times\left(\prod_{j=1}^{M^{\prime}}\left(1+O\left(\frac{s_{j} j}{M}\right)^{2}\right)\right) \\
& =M^{2 s} \sum_{l=1}^{M^{\prime}} \sum_{s_{1}+\ldots+s_{M^{\prime}}=s}\left(\prod_{j=1}^{M^{\prime}}\left(\begin{array}{c}
2 N-1+s_{j} \\
s_{j}
\end{array}\right)(2 j \pi)^{-2 s_{j}}\right) \\
& \times\left(O\left(\frac{s_{l} l}{M}\right)^{2} \prod_{j=1}^{l-1}\left(1+O\left(\frac{s_{j} j}{M}\right)^{2}\right)\right) \\
& +M^{2 s} \sum_{s_{1}+\ldots+s_{M^{\prime}}=s} \prod_{j=1}^{M^{\prime}}\left(\begin{array}{c}
2 N-1+s_{j} \\
s_{j}
\end{array}\right)(2 j \pi)^{-2 s_{j}} \\
& =M^{2 s}\left(\sum_{l=1}^{M^{\prime}} I_{l, M}(s)+A\left(M^{\prime}, s\right)\right)
\end{aligned}
$$

where $O\left(\frac{s_{j} j}{M}\right)^{2}$ denotes a term bounded by $C\left(\frac{s_{j} j}{M}\right)^{2}$ for some constant $C$ independent of 
M. Obviously, we have

$$
\begin{aligned}
0 & \leq I_{l, M}(s) \\
& \leq C \sum_{s_{l}=1}^{s}\left(\begin{array}{c}
2 N-1+s_{l} \\
s_{l}
\end{array}\right)(2 l \pi)^{-2\left(s_{l}-1\right)} M^{-2} \\
& \times \sum_{s_{1}+\ldots+s_{l-1}+s_{l+1}+\ldots+s_{M^{\prime}=s^{-} s_{l}}}\left(\prod_{j \neq l, 1 \leq j \leq M^{\prime}}\left(\begin{array}{c}
2 N-1+s_{j} \\
s_{j}
\end{array}\right)(2 j \pi)^{-2 s_{j}}\right) \\
& \leq C A\left(M^{\prime}, s\right) \sum_{s_{l}=1}^{s}(2 l \pi)^{-2\left(s_{l}-1\right)} M^{-2} \\
& \leq C M^{-2} A\left(M^{\prime}, s\right) .
\end{aligned}
$$

Hereafter the letter $C$ would denote a constant independent of $M$ which may be different at different instances. Therefore, we get $M_{N}^{-2 s} \underset{N}{M}(s) \rightarrow A_{s}$ as $M \rightarrow \infty$ when $M$ is odd.

When $M$ is even, we may write

$$
\begin{aligned}
& M^{-2 s} \stackrel{M}{N} a(s) \\
& =\sum_{s_{M / 2}=0}^{s}\left(\begin{array}{c}
N-1+s_{M / 2} \\
s_{M / 2}
\end{array}\right)(2 M)^{-2 s_{M / 2}} \\
& \times\left(M^{-2\left(s-s_{M / 2}\right)} \sum_{s_{1}+\ldots+s_{M / 2-1}=s-s_{M / 2}} \prod_{j=1}^{M / 2-1}\left(\begin{array}{c}
2 N-1+s_{j} \\
s_{j}
\end{array}\right)\left(2 \sin \frac{j \pi}{M}\right)^{-2 s_{j}}\right) \\
& =\sum_{s_{M / 2}=0}^{s}\left(\begin{array}{c}
N-1+s_{M / 2} \\
s_{M / 2}
\end{array}\right) 4^{-s_{M / 2}} M^{-2 s_{M / 2}} I\left(s-s_{M / 2}\right) \text {. }
\end{aligned}
$$

Using the same procedure to prove the assertion when $M$ is odd, we may prove that $I(t) \rightarrow A_{t}$ as $M \rightarrow \infty$ for all $0 \leq t \leq N-1$. Thus also $M_{N}^{-2 s} M a(s) \rightarrow A_{s}$ as $M \rightarrow \infty$ when $M$ is even

Lemma 3.2. Let ${ }_{N}^{M} a(N-1)$ be defined by (1.11). Then

$$
M^{-2 N+2} \underset{N}{M} a(N-1) \leq\left(1-\left(\frac{2}{\pi}\right)^{2 N}\right) 2^{-2(N-1)} .
$$

Proof. It is proved by $\mathrm{Bi}$ et al. in [1] that

$$
\sum_{s=0}^{M-1}\left|{ }_{N}^{M} H\left(\xi+\frac{2 \pi s}{M}\right)\right|^{2}=1 \text {. }
$$

We note that

$$
\sum_{s=0}^{M-1}\left|\frac{1-e^{i M \xi}}{M\left(1-e^{i(\xi+2 s \pi / M)}\right)}\right|^{2 N} \geq\left|\frac{\sin \frac{M \xi}{2}}{M \sin \frac{\xi}{2}}\right|^{2 N} \geq\left(\frac{2}{\pi}\right)^{2 N}
$$


when $|\xi| \leq \frac{\pi}{M}$. Therefore, we get

$$
\sum_{s=0}^{M-1}\left|\frac{1-e^{i M \xi}}{M\left(1-e^{i(\xi+2 s \pi / M)}\right)}\right|^{2 N} \geq\left(\frac{2}{\pi}\right)^{2 N} \quad(\xi \in \mathbb{R})
$$

We recall that

$$
\left|{ }_{N}^{M} H(\xi)\right|^{2}=\left(\frac{\sin \frac{M \xi}{2}}{M \sin \frac{\xi}{2}}\right)^{2 N} \sum_{s=0}^{N-1} 2^{2 s}{ }_{N} a(s)\left(\sin \frac{\xi}{2}\right)^{2 s}
$$

and $\underset{N}{M} a(0)=1$. Then it follows from (3.4) and (3.5) that

$$
\begin{aligned}
1-\left(\frac{2}{\pi}\right)^{2 N} \geq & M^{-2 N+2}{ }_{N}^{M} a(N-1)\left(\sin \frac{M \xi}{2}\right)^{2(N-1)} \\
& \times 2^{2(N-1)} \sum_{s=0}^{M-1} \frac{\sin ^{2} \frac{M \xi}{2}}{M^{2} \sin ^{2}\left(\frac{\xi}{2}+\frac{9 \pi}{M}\right)} \\
= & M^{-2 N+2}{ }_{N}^{M} a(N-1) 2^{2(N-1)}\left(\sin \frac{M \xi}{2}\right)^{2(N-1)}
\end{aligned}
$$

Substituting $\xi=\frac{\pi}{M}$ in (3.6) gives the lemma

Proof of Theorem 3.1. Let

$$
Q(t)=\sum_{s=0}^{N-1} A_{s} t^{s}
$$

By Lemma 3.1, we can write

$$
\widetilde{P}(t)=\sum_{s=0}^{N-1} N_{N}^{a} a(s) t^{s}=\sum_{s=0}^{N-1} \beta_{M}(s)\left(M^{2} t\right)^{s}
$$

where $\beta_{M}(s) \rightarrow A_{s}$ as $M \rightarrow \infty$. We then set

$$
Q(t)=\prod_{j=1}^{N-1}\left(\frac{t-t_{j}}{-t_{j}}\right)
$$

Then there exists a sequence $\left\{t_{j, M}\right\}_{j=1}^{N-1}$ such that

$$
\tilde{P}(t)=\prod_{j=1}^{N-1}\left(\frac{M^{2} t-t_{j, M}}{-t_{j, M}}\right)
$$

and $t_{j, M} \rightarrow t_{j}$ as $M \rightarrow \infty$. 
Recall that $\tilde{P}(t)>0$ when $t>0$. Therefore, $t_{j} \notin(0, \infty)$, and $t_{j, M} \notin(0, \infty)$ as $M$ is sufficiently large. When $t=2-e^{i \xi}-e^{-i \xi}$, we may write

$$
M^{2} t-t_{j, M}=\left[M\left(e^{i \xi}-1\right)-\theta_{j, M}\right]\left[M\left(e^{-i \xi}-1\right)-\theta_{j, M}\right] \times \beta_{j, M}
$$

where

$$
\left.\begin{array}{c}
\theta_{j, M}=\frac{-t_{j, M}-\sqrt{t_{j, M}^{2}-4 t_{j, M} M^{2}}}{2 M} \rightarrow-\sqrt{-t_{j}} \\
\beta_{j, M}=\frac{M}{M+\theta_{j, M}} \rightarrow 1
\end{array}\right\} \quad \text { as } M \rightarrow \infty .
$$

Furthermore, the real part of $\theta_{j, M}$ is always less than zero when $M$ is large enough. Therefore, the root of $M(z-1)-\theta_{j, M}$ is contained in the open unit disk and

$$
\prod_{j=1}^{N} \frac{M\left(e^{i \xi}-1\right)-\theta_{j, M}}{-\theta_{j, M}}
$$

is a trigonometrical polynomial with real coefficients. By the Riesz lemma [2: p. 172/Lemma 6.1.3] we obtain

$$
{ }_{N}^{M} \tilde{H}(\xi)=\prod_{j=1}^{N}\left[\frac{M\left(e^{i \xi}-1\right)-\theta_{j, M}}{-\theta_{j, M}}\right]
$$

Hence

$$
\lim _{M \rightarrow \infty} \sum_{s=0}^{N-1} M^{-s} a_{M}(s) t^{s}=\prod_{j=1}^{N-1}\left(\frac{t+\sqrt{-t_{j}}}{\sqrt{-t_{j}}}\right)
$$

and the $\operatorname{limit}_{\lim _{M \rightarrow \infty}} M^{-s} a_{M}(s)$ exists for all $0 \leq s \leq N-1$.

We observe that

$$
{ }_{N}^{M} H\left(\frac{\xi}{M}\right)=\left(\frac{1-e^{i \xi}}{M\left(1-e^{i \xi / M}\right)}\right)^{N} \sum_{s=0}^{N-1}\left(a_{M}(s) M^{-s}\right) \times\left(M\left(e^{i \xi / M}-1\right)\right)^{s}
$$

Then we find that

$$
\lim _{M \rightarrow \infty}{ }_{N}^{M} H\left(\frac{\xi}{M}\right)=\left(\frac{1-e^{i \xi}}{-i \xi}\right)^{N} \sum_{s=0}^{N-1} \alpha(s)(i \xi)^{s} .
$$

We recall from Lemma 3.2 that

$$
\prod_{j=1}^{N-1} \frac{1}{-t_{j, M}}=M^{-2(N-1)}{ }_{N}^{M} a(N-1) \leq 2^{-2(N-1)}\left(1-\left(\frac{2}{\pi}\right)^{2 N}\right) .
$$

Then we obtain

$$
\left|M^{-N+1} a_{M}(N-1)\right|=\left|\prod_{j=1}^{N} \frac{1}{\sqrt{-t_{j, M}}}\right| \leq 2^{-N+1}\left(1-\left(\frac{2}{\pi}\right)^{2 N}\right)^{1 / 2} .
$$

Theorem 3.1 is thus proved 


\section{Pointwise convergence and $L^{p}$-convergence}

The $B$-spline $B_{N}$ of degree $N-1$ is defined with the help of the Fourier transform by

$$
\widehat{B_{N}}(\xi)=\left(\frac{1-e^{i \xi}}{-i \xi}\right)^{N}
$$

Theorem 4.1. Let ${ }_{N}^{M} \phi$ be the solution of the refinement equation (1.1) with symbol ${ }_{N}^{M} H$ and $1 \leq p<\infty$. Then ${ }_{N}^{M} \phi$ converges pointwisely and in $L^{p}$-norm to

$$
g(x)=B_{N}(x)+\sum_{s=1}^{N-1} \alpha(s) B_{N}^{(s)}(x)
$$

where $\alpha(s)=\lim _{M \rightarrow \infty} M^{-s} a_{M}(s)$ and $B_{N}^{(s)}$ is the $s$-th derivative of $B_{N}$. Furthermore, $g$ is orthonormal.

We need the following lemmas to prove this theorem whose proof will be given later. For a compactly supported integrable function $f$, the $k$-moment of $f$ is defined by

$$
m_{k}(f)=\int_{\mathbb{R}} x^{k} f(x) d x \quad(0 \leq k \leq N-1) .
$$

Then we have the following

Lemma 4.1. Let ${ }_{N}^{M} \phi$ be the solution of the refinement equation (1.1) with symbol $\stackrel{M}{N}$. Then

$$
\lim _{M \rightarrow \infty} m_{k}(\stackrel{M}{N} \phi)=m_{k}\left(B_{N}\right)+\sum_{j=1}^{N-1} \alpha(j) m_{k}\left(B_{N}^{(j)}\right) \quad(0 \leq k \leq N-1)
$$

holds.

Proof. Let $D=i \frac{d}{d \xi}$ be a differential operator. Then for any compactly supported integrable function $f$, we have $m_{k}(f)=\left(D^{k} \widehat{f}\right)(0)$. Define

$$
h_{M}(\xi)=\prod_{j=1}^{\infty}{ }_{N}^{M} \widetilde{H}\left(\frac{\xi}{M^{j}}\right)
$$

Then we have

$$
h_{M}(\xi)={ }_{N}^{M} \widetilde{H}\left(\frac{\xi}{M}\right) h_{M}\left(\frac{\xi}{M}\right)
$$

and

$$
D^{k} h_{M}(\xi)=M^{-k} \sum_{j=0}^{k}\left(\begin{array}{l}
k \\
j
\end{array}\right)\left(D_{N}^{j M} \widetilde{H}\right)\left(\frac{\xi}{M}\right)\left(D^{k-j} h_{M}\right)\left(\frac{\xi}{M}\right)
$$


Hence, we find

$$
\left(1-M^{-k}\right)\left(D^{k} h_{M}\right)(0)=M^{-k} \sum_{j=1}^{k}\left(\begin{array}{l}
k \\
j
\end{array}\right)\left(D_{N}^{j M} \tilde{H}\right)(0)\left(D^{k-j} h_{M}\right)(0)
$$

From Theorem 3.1, $M^{-j}\left(D^{j} \underset{N}{M} \widetilde{H}\right)(0) \rightarrow(-1)^{j} j ! \alpha(j)$ as $M \rightarrow \infty \quad(0 \leq j \leq N-1)$. Hence $D^{k} h_{M}(0) \rightarrow(-1)^{k} k ! \alpha(k)$ as $M \rightarrow \infty(0 \leq k \leq N-1)$.

We recall that

$$
{ }_{N}^{M} H(\xi)=\left(\frac{1-e^{i M \xi}}{M\left(1-e^{i \xi}\right)}\right)^{N} \stackrel{M}{N} \tilde{H}(\xi)
$$

Therefore, $\widehat{M} \phi(\xi)=\widehat{B_{N}}(\xi) h_{M}(\xi)$, and

$$
\left(D_{N}^{k} \widehat{M} \phi\right)(0)=\sum_{j=0}^{k}\left(\begin{array}{l}
k \\
j
\end{array}\right)\left(D^{j} \widehat{B_{N}}\right)(0)\left(D^{k-j} h_{M}\right)(0)
$$

Hence

$$
\left(D^{k} \widehat{M} \phi\right)(0) \rightarrow \sum_{j=0}^{k} \frac{k !}{(k-j) !}(-1)^{j} \alpha(j) D^{k-j} \widehat{B_{N}}(0)=\sum_{j=0}^{N-1} \alpha(j) \int_{\mathbb{R}} x^{k} B_{N}^{(j)}(x) d x
$$

and the lemma is proved

Lemma 4.2. Let $d_{j, M}(0 \leq j \leq N-1)$ be numbers such that

$$
\stackrel{M}{N}^{M}\left(\frac{x+j}{M}\right)=d_{j, M} \stackrel{M}{N} \phi(x)+Q_{j, M}(x) \quad(x \in(0,1), 0 \leq j \leq N-1)
$$

holds for a polynomial $Q_{j, M} \in \Pi_{N-1}$. Then

$$
\beta_{j}=\lim _{M \rightarrow \infty} d_{j, M}=(-1)^{N-1} \alpha(N-1) \sum_{s=0}^{j} \frac{(-1)^{s} N !}{s !(N-s) !}
$$

and

$$
\left|\beta_{j}\right| \leq 2^{N-1}|\alpha(N-1)| \leq\left(1-\left(\frac{2}{\pi}\right)^{2 N}\right)^{1 / 2} \quad(0 \leq j \leq N-1) .
$$

Proof. We recall that

$$
\sum_{k \in \mathbf{Z}}(x+k)_{N}^{j M} \phi(x+k)=m_{j}\left(\begin{array}{l}
M \\
N
\end{array}\right) \quad(0 \leq j \leq N-1) .
$$

Then we obtain

$$
\sum_{k \in \mathbf{Z}} k_{N}^{j} \phi(\cdot+k)=Q ; \in \Pi_{N-1} \quad(0 \leq j \leq N-1)
$$


or, in matrix form,

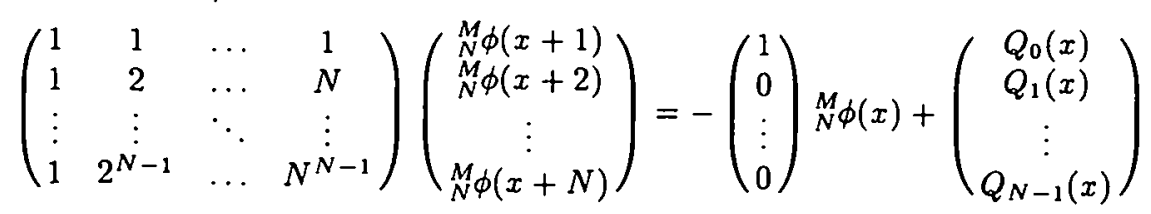

on $(0,1)$. Therefore, we get

$$
{ }_{N}^{M} \phi(x+j)=\frac{(-1)^{j} N !}{j !(N-j) !} \stackrel{M}{N} \phi(x)+\tilde{Q}_{j}(x)
$$

where $\widetilde{Q}_{j} \in \Pi_{N-1}$. It follows from Theorem 3.1 that

$$
\begin{aligned}
{ }_{N}^{M} H(\xi) & =\left(\frac{e^{i M \xi}-1}{M\left(e^{i \xi}-1\right)}\right)^{N} \sum_{s=0}^{N-1} a_{M}(s)\left(e^{i \xi}-1\right)^{s} \\
& =\frac{1}{M} \alpha(N-1)\left(\frac{1-e^{i M \xi}}{1-e^{i \xi}}\right)\left(e^{i M \xi}-1\right)^{N-1}+\frac{1}{M} \sum_{k=0}^{M N-1} o(1) e^{i k \xi}
\end{aligned}
$$

where $o(1)$ means a number tending to zero as $M \rightarrow \infty$. From (2.4) and (4.4), there exist polynomials $Q_{j} \in \Pi_{N-1}$ such that

$$
\begin{aligned}
\stackrel{M}{N} \phi_{\left(\frac{x+j}{M}\right)} & =\sum_{l=0}^{j} c_{j-l}^{M} \phi(x+l) \\
& =(-1)^{N-1} \alpha(N-1) \sum_{l=0}^{j}(1+o(1))_{N}^{M} \phi(x+l) \\
& =\left((-1)^{N-1} \alpha(N-1) \sum_{l=0}^{j}(-1)^{l}\left(\begin{array}{c}
N \\
l
\end{array}\right)\right){ }_{N}^{M} \phi(x)+o(1)_{N}^{M} \phi(x)+Q_{j}(x) .
\end{aligned}
$$

This proves the first assertion.

Observe that $\sum_{s=0}^{N}(-1)^{s}\left(\begin{array}{c}N \\ s\end{array}\right)=0$. Then we have

$$
\left|\sum_{s=0}^{j}(-1)^{s}\left(\begin{array}{c}
N \\
s
\end{array}\right)\right|=\left|\sum_{s=0}^{N-j-1}(-1)^{s}\left(\begin{array}{c}
N \\
s
\end{array}\right)\right| .
$$

It is easy to see that

$$
\left|\sum_{s=0}^{j}(-1)^{s}\left(\begin{array}{c}
N \\
s
\end{array}\right)\right| \leq\left(\begin{array}{c}
N \\
j
\end{array}\right)
$$

when $j<\frac{N}{2}$. Then from the identity $2^{N}=(1+1)^{N}=\sum_{s=0}^{N}\left(\begin{array}{c}N \\ s\end{array}\right)$ we get $\left(\begin{array}{c}N \\ (N-1) / 2\end{array}\right) \leq$ $2^{N-1}$ when $N$ is odd and $\left(\begin{array}{c}N \\ N / 2-1\end{array}\right) \leq 2^{N-1}$. Observe that

$$
\left(\begin{array}{c}
N \\
j
\end{array}\right) \leq\left\{\begin{array}{ll}
\left(\begin{array}{c}
N \\
(N-1) / 2
\end{array}\right) & \text { when } N \text { is odd } \\
\left(\begin{array}{c}
N \\
N / 2-1
\end{array}\right) & \text { when } N \text { is even. }
\end{array} \quad\left(0 \leq j<\frac{N}{2}\right) .\right.
$$

Thus, the second assertion follows from Theorem 3.1 
Proof of Theorem 4.1. It follows from (2.1) that

$$
A(x)_{N}^{M} \Phi(x)=\left(m_{0}(\stackrel{M}{N} \phi), \ldots, m_{N-1}(\stackrel{M}{N} \phi)\right)^{T} \quad \text { on }\left(\frac{N-1}{M-1}, 1\right)
$$

where ${ }_{N}^{M} \Phi(x)=(\stackrel{M}{N} \phi(x), \ldots, \stackrel{M}{N} \phi(x+N-1))$. Recall that

$$
m_{k}(\stackrel{M}{N} \phi) \rightarrow \sum_{j=0}^{N-1} \alpha(j) m_{k}\left(B_{N}^{(j)}\right)
$$

by Lemma 4.1. Therefore,

$$
{ }_{N}^{M} \Phi(x) \rightarrow \sum_{j=0}^{N-1} \alpha(j) A^{-1}(x)\left(m_{0}\left(B_{N}^{(j)}\right), \ldots, m_{N-1}\left(B_{N}^{(j)}\right)\right)^{T}
$$

pointwisely on $(0,1)$. This proves that

$$
\stackrel{M}{N} \phi(x) \rightarrow g(x)=\sum_{j=0}^{N-1} \alpha(j) B_{N}^{(j)}(x)
$$

Obviously, by the dominated convergence theorem, the $L^{p}$-convergence $(1 \leq p<\infty)$ of $\stackrel{M}{N} \phi(x)$ reduces to prove that ${ }_{N}^{M} \phi(x)$ is uniformly bounded. Recall that

$$
{ }_{N}^{M} \phi\left(\frac{x+j}{M}\right)=d_{j, M} \stackrel{M}{N} \phi(x)+Q_{j, M}(x) \quad \text { and } \quad\left|d_{j, M}\right| \leq\left(1-2\left(\frac{2}{\pi}\right)^{2 N}\right)^{1 / 2}
$$

when $M$ is sufficiently large and $Q_{j, M}$ is uniformly bounded by $C$. Thus we get

$$
\begin{aligned}
\sup _{x \in A\left(e_{1}, \ldots, \varepsilon_{k}\right)}\left|{ }_{N}^{M} \phi(x)\right| & \leq C\left(1-2\left(\frac{2}{\pi}\right)^{2 N}\right)^{\frac{1}{2} k}+C \sum_{j=0}^{k-1}\left(1-2\left(\frac{2}{\pi}\right)^{2 N}\right)^{\frac{1}{2} j} \\
& \leq C\left(\frac{\pi}{2}\right)^{2 N}
\end{aligned}
$$

It follows from the proof of Theorem 2.1 that

$$
\bigcup_{k=1}^{\infty} \bigcup_{\substack{\varepsilon_{i} \in\{0,1, \ldots, N-1\}, 1 \leq i \leq k-1 \\ \varepsilon_{k} \in\{0,1 \ldots, N-2\}}} A\left(\varepsilon_{1}, \ldots, \varepsilon_{k}\right) \cup\left(\frac{N-1}{M-1}, 1\right)
$$

has Lebesgue measure 1 . This proves that ${ }_{N}^{M} \phi$ is uniformly bounded. Recall that ${ }_{N}^{M} \phi$ is orthonormal. Then the limit $g$ of ${ }_{N}^{M} \phi$ in the $L^{2}$-norm is also orthonormal 


\section{The limit function}

In this section we will give a method to construct the limit function $g$ in Theorem 4.1. Let

$$
G(z)=\sum_{s=0}^{N-1} \alpha(s) z^{s} \quad \text { and } \quad Q(z)=\sum_{s=0}^{N-1} A_{s} z^{2 s}
$$

Then, by the proof of Theorem 3.1, we get

$$
G(i z) G(-i z)=Q(z) \text {. }
$$

Let $g$ be the limit of ${ }_{N}^{M} \phi$ in Theorem 4.1. Then $g$ is unique determined by $G$.

Now we compute $A_{s}(0 \leq s \leq N-1)$ explicitly. Observe that $g$ is orthonormal by Theorem 4.1. Then we have

$$
\sum_{k \in \mathbf{Z}}|\hat{g}(\xi+2 k \pi)|^{2}=1 \quad(\xi \in \mathbb{R}) .
$$

By Theorem 4.1 and by the orthonormality of $\stackrel{M}{N} \phi$, we get $\hat{g}(\xi)=G(i \xi) \widehat{B_{N}}(\xi)$ and

$$
\sum_{k \in \mathbf{Z}} Q(\xi+2 k \pi)\left|\widehat{B_{N}}(\xi+2 k \pi)\right|^{2}=1 \quad \text { for all } \xi \in \mathbb{R} \text {. }
$$

Let $\widetilde{B}_{2 N}(x)=B_{2 N}(x+N)$. Then $\widehat{\widetilde{B}_{2 N}}(\xi)=\left|\widehat{B}_{N}(\xi)\right|^{2}$ and the function $\tilde{g}$ defined by $\tilde{g}(x)=\sum_{s=0}^{N-1} A_{s} \widehat{B_{2 N}^{(2 g)}}(x)$ satisfies the interpolation condition, that means $\tilde{g}$ takes the value zero at integer lattice except $\tilde{g}(0)=1$. Hence $A_{s}$ satisfies the equation

$$
\left(\begin{array}{cccc}
\tilde{B}_{2 N}(0) & \tilde{B}_{2 N}^{\prime \prime}(0) & \ldots & \tilde{B}_{2 N}^{(2 N-2)}(0) \\
\tilde{B}_{2 N}(1) & \tilde{B}_{2 N}^{\prime \prime}(1) & \ldots & \tilde{B}_{2 N}^{(2 N-2)}(1) \\
\vdots & \vdots & \ddots & \vdots \\
\tilde{B}_{2 N}(N-1) & \tilde{B}_{2 N}^{\prime \prime}(N-1) & \ldots & \tilde{B}_{2 N}^{(2 N-2)}(N-1)
\end{array}\right)\left(\begin{array}{c}
A_{0} \\
A_{1} \\
\vdots \\
A_{N-1}
\end{array}\right)=\left(\begin{array}{c}
1 \\
0 \\
\vdots \\
0
\end{array}\right)
$$

The above equation can be solved by the following iterative algorithm.

\section{ALGORITHM:}

Step 1. Define $B(s, \xi)=\sum_{k \in \mathbf{Z}} \widetilde{B}_{2 N-2 s}(k) e^{i k \xi}$ and $G_{0}(\xi)=1$.

Step 2. Define $A_{s}=G_{s}(0)$.

Step 3. Define $G_{s+1}(\xi)=\left(2-e^{-i \xi}-e^{i \xi}\right)^{-1}\left(G_{s}(\xi)-A, B(s, \xi)\right)$.

Step 4. Return to Step 2 if $s \leq N-2$ and stop if $s=N-1$.

From the above equation, we see that the solution of equation (5.2) is unique, and it is just equal to $A_{9}$. This gives explicit description of $A_{\text {, where }} 0 \leq s \leq N-1$.

Now we can show how to construct the coefficient $\alpha(s)$. First, we write

$$
Q(z)=\sum_{s=0}^{N-1} A_{s} z^{2 s}=\prod_{j=0}^{N-1}\left(\frac{z^{2}-t_{j}}{-t_{j}}\right) .
$$

Then $\alpha(s)$ satisfies

$$
\sum_{s=0}^{N-1} \alpha(s) z^{s}=\prod_{j=0}^{N-1}\left(\frac{z+\sqrt{-t_{j}}}{\sqrt{-t_{j}}}\right) .
$$

This give a explicit construction of $g$ in Theorem 4.1. 
Remark 1. From Theorem 3.1, we see that

$$
\lim _{M \rightarrow \infty}{ }_{N}^{M} H\left(\frac{\xi}{M}\right)=\left(\frac{1-e^{i \xi}}{-i \xi}\right)^{N} \cdot \sum_{s=0}^{N-1} \alpha(s)(i \xi)^{s}=\hat{g}(\xi)
$$

Therefore, $\widehat{N} \phi(\xi) \rightarrow \hat{g}(\xi)$ uniformly on any bounded set.

Remark 2. Observe that the solution of equation (5.1) is not unique. In particular, the polynomial

$$
\tilde{Q}(z)=\prod_{j=0}^{N-1}\left(\frac{z \pm \sqrt{-t_{j}}}{ \pm \sqrt{-t_{j}}}\right)
$$

also satisfies equation (5.1). After careful choice of positive or negative sign in (5.4), we can make $\tilde{Q}$ to be a polynomial with real coefficients. Using the method of Theorem 4.1 , we may find a class of scaling functions ${ }_{N}^{M} \tilde{\phi}$ with the symbol

$$
{ }_{N}^{M} \tilde{H}(\xi)=\left(\frac{1-e^{i M \xi}}{M\left(1-e^{i \xi}\right)}\right)^{N} \sum_{s=0}^{N-1} \tilde{a}_{M}(s) e^{i \xi}
$$

satisfying equation (1.8) such that its limit function is $\sum_{s=0}^{N-1} \tilde{\alpha}(s) B_{N}^{(s)}(x)$ where $\tilde{Q}(z)=$ $\sum_{s=0}^{N-1} \tilde{\alpha}(s) z^{s}$.

\section{References}

[1] Bi, N., Dai, X. and Q. Sun: Construction of compactly supported $M$-band wavelets. Report. Zhejiang (China): Center Math. Sci. Univ., Research Report 9518 (1995); pp. 1 25.

[2] Daubechies, I.: Ten Lectures on Wavelets (CBMS-NSF Regional Conference Series in Applied Mathematics: Vol. 61). Philadelphia: SIAM 1992.

[3] Heller, P. N.: Rank $M$ wavelets with $N$ vanishing moments. SIAM J. Matrix Anal. 16 (1995), $502-519$.

[4] Pollen, D.: Daubechies' scaling function on [0,3]. In: Wavelet - A Tutorial in Theory and Application (ed.: C. K. Chui). Boston: Academic Press Inc. 1992, pp. 3 - 13.

[5] Sun, Q. and Z. Zhang: $M$-band scaling function with filter having vanishing moments two and minimal length. Preprint, Sept. 1996.

Received 25.09.1997; in revised form 23.09.1998 